\title{
VALIDITY OF GENERATION SYSTEM FOR SOLITARY WAVE BOUNDARY LAYER
}

\author{
Bambang WINARTA ${ }^{1}$ and Hitoshi TANAKA ${ }^{2}$ \\ ${ }^{1}$ Student Member of JSCE, M. Eng., Graduate School of Eng., Dept. of Civil Engineering, Tohoku University \\ (6-6-06 Aoba, Sendai 980-8579, Japan) \\ ${ }^{2}$ Fellow Member of JSCE, Dr. of Eng., Professor, Dept. of Civil Engineering, Tohoku University \\ (6-6-06 Aoba, Sendai 980-8579, Japan)
}

\begin{abstract}
The present study is concerning bottom boundary layer beneath solitary wave over smooth beds condition. A new generation system was proposed to generate an oscillatory motion similar to solitary wave in a closed conduit water tunnel using a mechanical system. This generation system facilitates easy measurement of periodical oscillatory motion to replace solitary wave motion with a sufficient tranquil period. The velocities were measured by using a Laser Doppler Veloci-meter (LDV) at 17 to 22 points in the vertical direction. The experiments were accomplished with different velocities under single and periodical oscillatory motion conditions and validations have been done in some various terms of experiment relevant to solitary wave boundary layer. A good agreement is achieved in validation of free stream velocity and also both single and periodical oscillatory motion measurements methods. Furthermore, validation of velocity distribution in time variation obtains a critical Reynolds number which has a good agreement with the finding of previous researchers.
\end{abstract}

Key Words : Solitary wave, bottom boundary layer, generation system, validation, transition to turbulence

\section{INTRODUCTION}

Laboratory and numerical experiments of bottom boundary layer under non-linear wave motion has been done by many researchers. However, only several researchers have conducted experimental and theoretical studies to describe the wave boundary layer under solitary wave motion.

Laboratory experiments using a wave flume have been done by Liu et al. ${ }^{1}$ to investigate the boundary layer characteristics under solitary wave. Their experimental system is assisted by Particle Image Velocimetri (PIV) to measure velocity in the thin boundary layer. According to their experimental result, it is concluded that the experiments fall in laminar regime and still cannot investigate in transitional and turbulent regimes.

Later, an oscillatory flow generator to scrutinize bottom boundary layer under solitary wave have been proposed by Konishi et al. ${ }^{2)}$ but their experiment results of bottom shear stress and vertical velocity distribution different from numerical model conducted by the baseline $k-\omega$ turbulent model. Yamaji et al. ${ }^{3)}$ employed similar generation system using a closed conduit water tunnel facilitated by movable gate. It was controlled by mechanism of rotating disc with combination of semi-circle and half ellipse as rotating disc shape. An excellent agreement was achieved between theory and experiment in term of free stream velocity. However, the experimental velocity distribution has negative value during the early stage of oscillatory motion, which is caused by the preceding periodical motion.

Recently, laboratory experiments to observe turbulent solitary wave boundary layer have been accomplished by Sumer et al. ${ }^{4}$. Their experimental result showed that Reynolds number $\left(R_{e}\right)$ up to $1 \times 10^{6}$ is still in laminar flow regime. A U-shape oscillating water tunnel was used to investigate wave boundary layer characteristics induced by solitary wave motion. However, it will be practically difficult to generate boundary layer flow corresponds to solitary wave motion precisely in their facility because of restorative force in tunnel, which may induce oscillating motion with flow reversal. Indeed in their experimental result analysis, the portion of the data with negative velocities at the trailing of the fluid motion was ignored. Therefore, a new generation method which enables generation 
of boundary layer flow under solitary wave is highly required.

In the present study, a new generation method equipped by the new design of rotating disc based on solitary wave theory has been applied to examine boundary layer characteristics under solitary wave over smooth bed condition and then validation has been done for free stream velocity, single and periodical oscillatory motion measurements and also for time variation of velocity distribution in order to identify transition to turbulence.

\section{THEORITICAL BACKGROUND AND EXPERIMENTAL SYSTEM}

\section{(1) Solitary wave}

The free stream velocity under solitary wave is given as

$$
U=U_{c} \sec h^{2}(\xi)
$$

where $U_{c}$ is the maximum velocity under wave crest and $\xi$ is defined by

$$
\xi=\alpha t
$$

in which $t$ is the time and

$$
\alpha=\sqrt{\frac{3 H}{4 h^{3}}} \sqrt{g(h+H)}
$$

where $h$ : the water depth, $H$ : the wave height and $g$ : the gravitational acceleration.

An expression for the horizontal velocity inside the boundary layer has been derived by Keulegan ${ }^{5}$, considering a linearized boundary layer equation and laminar flow condition. Later, Keulegan's expression has been converted to temporal variation as follows by Tanaka et al. ${ }^{6}$.

$\frac{u}{U_{c}}=\operatorname{sech}^{2}(\xi)-\frac{2}{\sqrt{\pi}} \int_{0}^{\infty} \operatorname{sech}^{2}\left\{\xi+\left(\frac{\beta^{\prime} z}{\beta}\right)^{2}\right\} e^{-\beta^{2}} d \beta$

where $u$ : the horizontal velocity in the boundary layer, $z$ : the vertical distance from the bottom and $\beta$, is defined as

$$
\beta^{\prime}=\frac{1}{2} \sqrt{\frac{a_{S} c}{v}}
$$

in which $v$ : the kinematic viscosity of fluid and $a_{s}$ is defined as

$$
a_{s}=\sqrt{\frac{3 H}{4 h^{3}}}
$$

\section{(2) Experimental system}

A general sketch of the experiment set-up used in this study is given in Fig. 1(a). It is consists of an overflow head tank, downstream gate, a LDV and a conduit water tunnel.

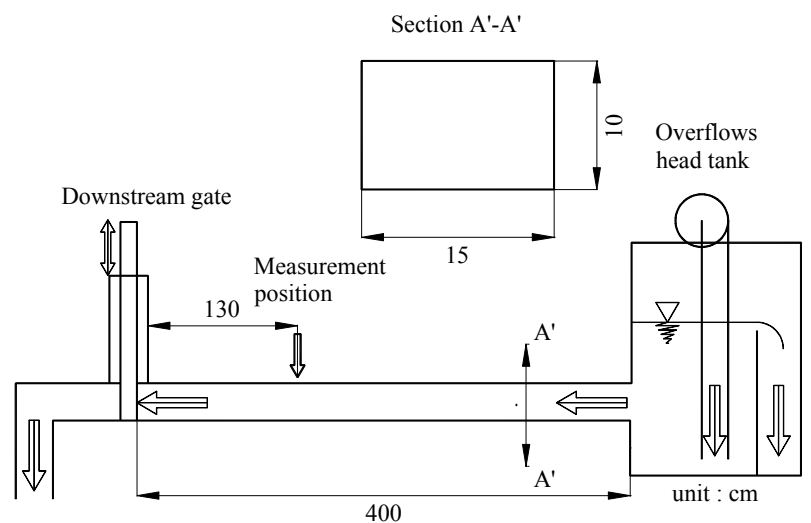

(a)

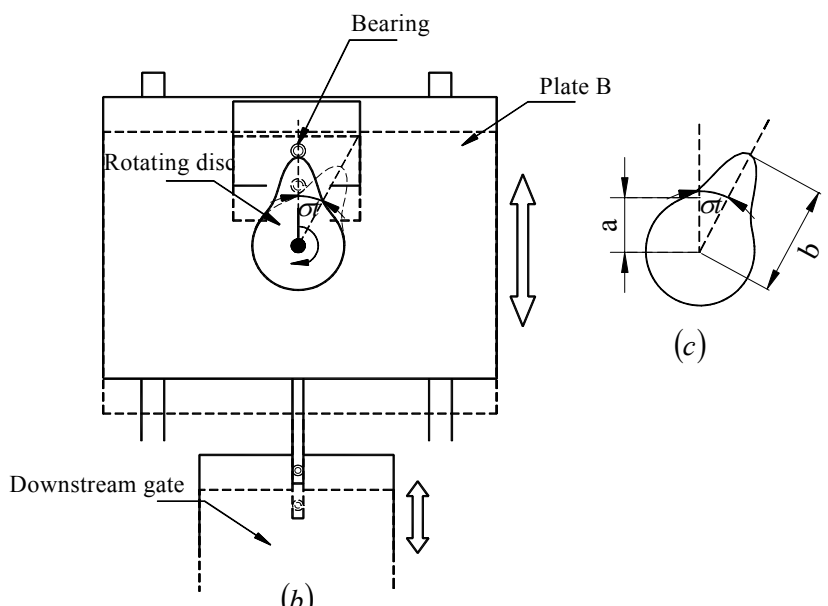

(b)

Fig. 1(a) Front view of generation system sketch; (b) mechanism of rotating disc and (c) rotating disc design

The overflow head tank keeps a constant pressure head and then water flows into measurement section intermittently along a conduit part in response to opening and closing of the gate controlled by rotating disc mechanism. The mechanism of a rotating disc for flow generating is illustrated in Fig. 1(b) and the rotating disc shape shown in Fig. 1(c). The downstream gate is completely closed during attachment of the bearing to semi-circle portion, while it will opens gradually and subsequently closes when it attaches the rest of the portion of the disc. The time-variation of horizontal velocity in a closed conduit can be expressed as follows, considering the exact solution of the solitary wave theory given by Eq.(1).

$$
\frac{U(t)}{U_{c}}=\frac{z_{g}(t)}{z_{g \max }}=\operatorname{sech}^{2}(\gamma \sigma t)
$$

where $U(t)$ is the horizontal velocity in the closed conduit, $z_{g}(t)$ is the height of opening the gate, $\gamma$ is the parameter to control the length of tranquil period and the subscript max denotes the maximum value. Therefore, the corresponding expression for the shape of the rotating disc is given the following equation.

$$
z_{d}(t)=a+z_{g \max } \operatorname{sech}^{2}(\gamma \sigma t)
$$




$$
\begin{aligned}
& \text { for }-\pi / 2<\sigma t<\pi / 2 \\
& z_{d}(t)=a \\
& \quad \text { for }-\pi<\sigma t<-\pi / 2, \pi / 2<\sigma t<\pi
\end{aligned}
$$

where $z_{d}(t)$ is the distance from the center of the disc to the edge at $\sigma t$ and $\sigma$ is angular frequency of a rotating disc. Thus, $U(t)$ becomes the maximum velocity $U_{c}$ when the height of opening gate is maximum at $\sigma t=0$. It should be here noted that the flow motion generated by this generation system is not purely "solitary" but periodical consists "solitary wave like" positive peaks and tranquil period in between two peaks.

According to Eq. (1) and Eq. (2), it takes infinite time for velocity to reach $U=0$. For practical purposes, however, the time required for substantial decay of velocity can be defined such that $\Delta U^{*}=$ $U(\sigma t=\pi / 2) / U_{c}=0.01,0.001$ etc. The relationship between $\Delta U^{*}$ and $\gamma$ is obtained from Eq. (7), as shown below

$$
\gamma=\frac{2}{\pi} \operatorname{sech}^{-1}\left(\sqrt{\Delta U^{*}}\right)
$$

And the relationship between $\alpha$ and $\gamma$ can be obtained from Eqs. (1), (2), and (7), as written below

$$
\alpha=\gamma \sigma
$$

In the present experiment rotating disc was design by using $\Delta U^{*}=0.001$ and $\gamma$ will be equal to 2.630 .

\section{EXPERIMENTAL CONDITION}

Laboratory experiments have been done for various maximum velocities under single and continuous (or periodical) oscillatory motion. Single and periodical oscillatory motion measurements were carried out for Case 2, 3 and 4, whereas Case 1 was only under periodical oscillatory motion. The single oscillatory motion measurement has purposes to evaluate the sufficiency of tranquil period between two peaks of periodical motion. The present experimental conditions are summarized in Table 1 and the solitary wave $R_{e}$ is defined by the following equation based on $U_{c}$ and $\alpha^{9)}$.

$$
R_{e}=\frac{U_{c}^{2}}{v \alpha}
$$

Table 1 Experimental condition

\begin{tabular}{|c|c|c|c|c|}
\hline & $v\left(\mathrm{~cm}^{2} / \mathrm{s}\right)$ & $U_{c}(\mathrm{~cm} / \mathrm{s})$ & $\alpha\left(\mathrm{s}^{-1}\right)$ & $R_{e}$ \\
\hline Case 1 & 0.0100 & 42.6 & 0.79 & $2.25 \times 10^{5}$ \\
\hline Case 2 & 0.0116 & 78.7 & 0.95 & $5.64 \times 10^{5}$ \\
\hline Case 3 & 0.0116 & 78.5 & 0.88 & $6.06 \times 10^{5}$ \\
\hline Case 4 & 0.0114 & 81.3 & 0.81 & $7.34 \times 10^{5}$ \\
\hline
\end{tabular}

\section{EXPERIMENTAL RESULTS}

\section{(1) Time variation of free stream velocity}

The free stream velocity obtained from present experiment is compared with theory for all cases as shown in Fig. 2. From those figures, it is concluded that good agreement can be achieved, although there is a slight different especially in the early of periodical motion. However, by comparing with the previous experimental used by Konishi et al. ${ }^{3)}$ and Yamaji et al. ${ }^{4}$, the present experiment gives much better result for the free stream velocity. Further, it should be here emphasized that the negative velocity at the trailing of fluid motion inherent in a U-shape oscillating tunnel as reported by Sumer et al. ${ }^{4)}$ can be definitely avoided.

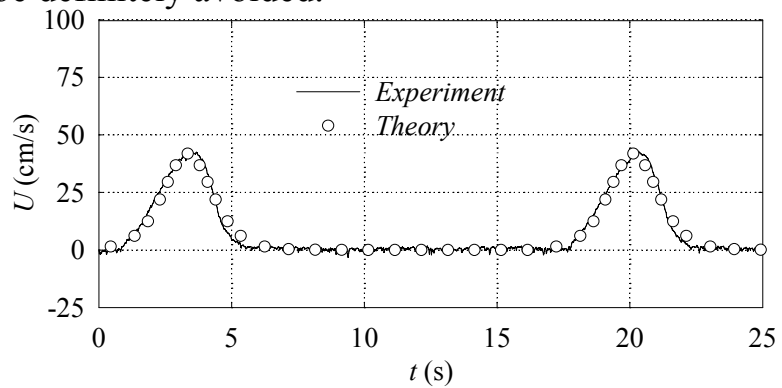

(a) Case $1\left(R_{e}=2.25 \times 10^{5}\right)$

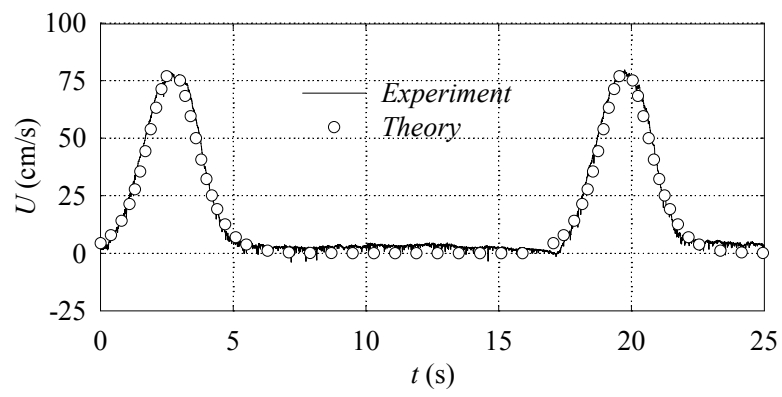

(b) Case $2\left(R_{e}=5.64 \times 10^{5}\right)$

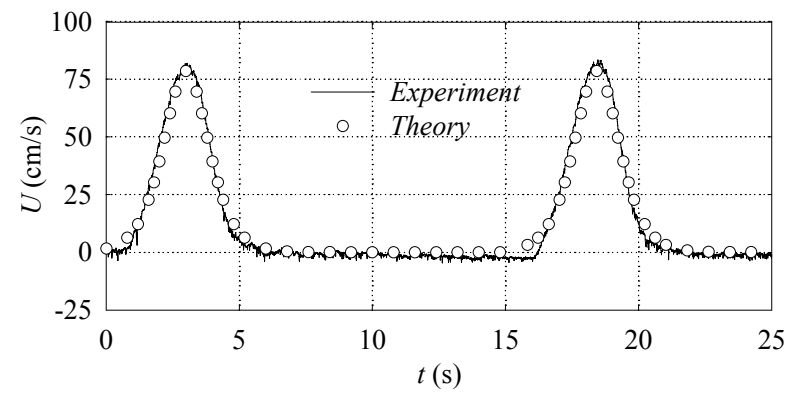

(c) Case $3\left(R_{e}=6.06 \times 10^{5}\right)$

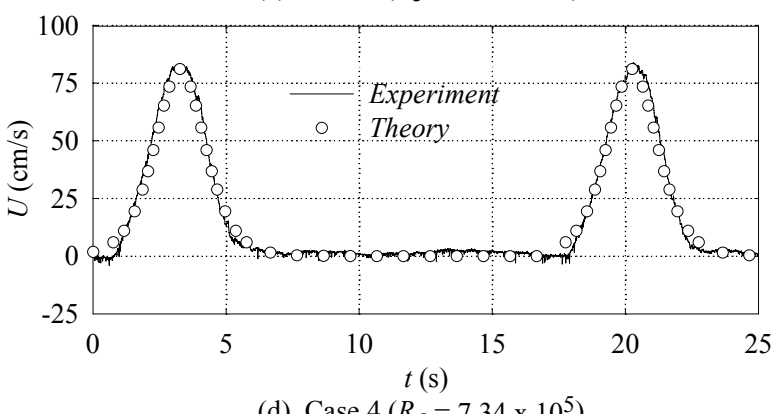

Fig. 2 Time variation of free stream velocity 


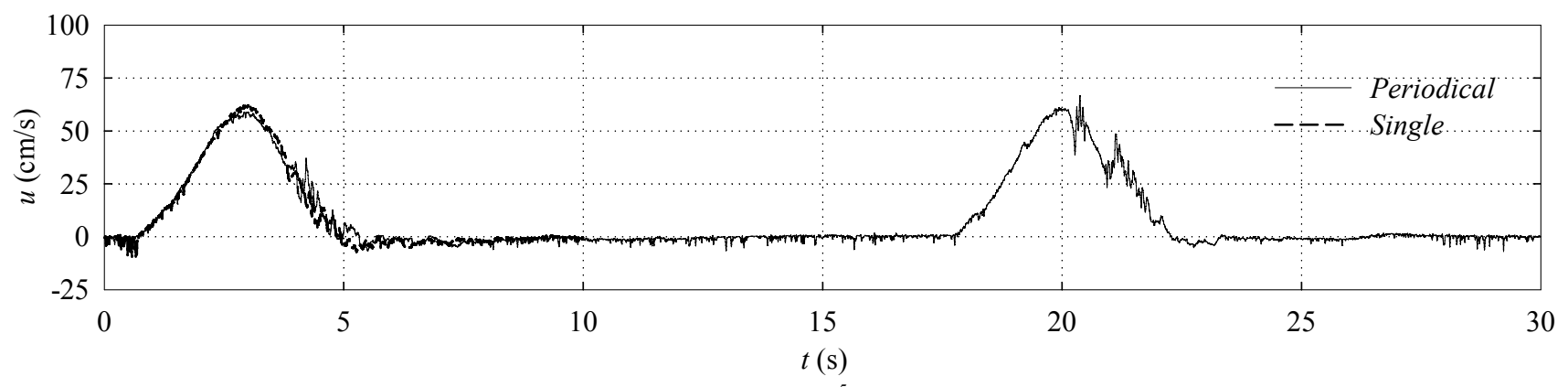

(a) $R_{e}=7.34 \times 10^{5} ; z=0.15 \mathrm{~cm}$

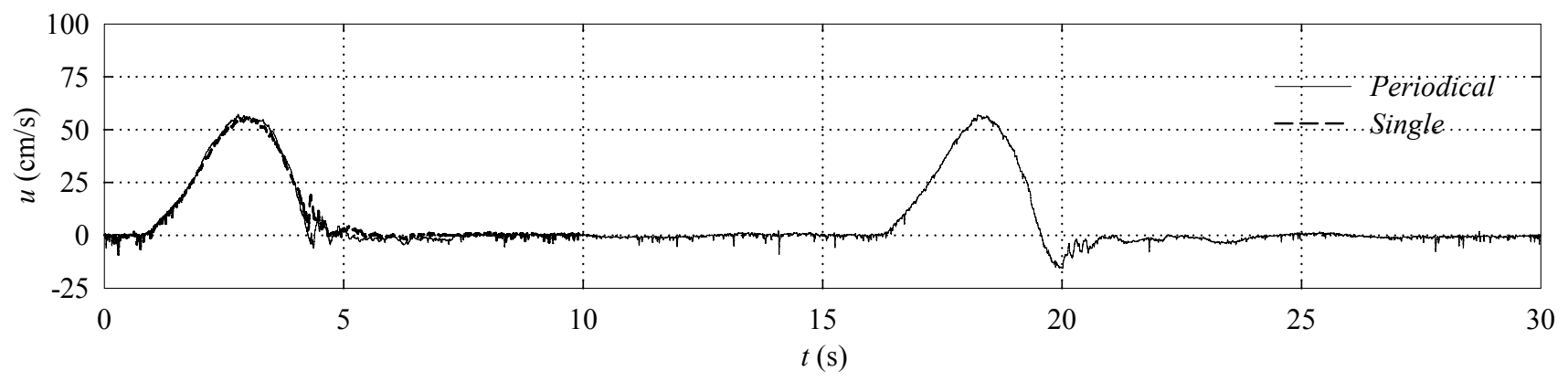

(b) $R_{e}=6.06 \times 10^{5} ; z=0.151 \mathrm{~cm}$

Fig. 3 Single and periodical oscillatory motion measurement

(2) Comparison between single and periodical oscillatory motion measurements

As mentioned before, the present laboratory experiment has been performed for both generation methods under single and periodical oscillatory motion. Figure 3 illustrates the comparison between two kinds of measurement method for two measurement points in the vertical direction. As a conclusion, single and periodical oscillatory motion measurements give a good agreement, indicating validity of the periodical generation system. Thus, from a view point of efficient laboratory efficient laboratory experiment, the generation system with periodical generation is useful for various kinds of experiment relevant to solitary wave boundary layer.

\section{(3) Transition to turbulence}

In order to validate the generation method, the behavior of transition to turbulence will be discussed based on the laboratory data. Figure 4 shows the comparison of horizontal velocity measurement obtained from present experiment and laminar solution at two elevations in the vicinity to the wall for all cases. Figure 4(a) shows comparison both of them in the lowest $R_{e}$ (Case 1; $R_{e}=2.25 \times 10^{5}$ ) and Fig. 4(d) in the highest $R_{e}$ (Case 4; $R_{e}=7.34 \times 10^{5}$ ).

According to Hino et al. ${ }^{8}$, turbulent flow regime can be classified in three types: weakly turbulent flow, conditionally turbulent flow and fully turbulent flow. They defined conditionally turbulent flow when turbulence is generated suddenly in the decelerating phase while the flow recovers to laminar in the accelerating phase. This phenomenon can be observed in the Case 3 and Case 4 with $R_{e}=$ $7.34 \times 10^{5}$ and $R_{e}=6.06 \times 10^{5}$ respectively. The horizontal velocity distributions for these two cases are shown in Fig. 4(c) and Fig. 4(d). From those figures can be seen clearly that turbulence or spike is generated suddenly in the decelerating phase, while in the accelerating phase, the flow coincide well with laminar solution. It can be concluded that Case 3 and Case 4 are fall in conditionally turbulent flow.

Different characteristic can be observed apparently in the lowest $R_{e}$ case as drawn in Fig. 4(a), excellent agreement is achieved between laminar solution and experimental result in a horizontal velocity distribution and it can be concluded as laminar flow regime (Case $1, R_{e}=$ $\left.2.25 \times 10^{5}\right)$. A small fluctuation occurred during periodical motion. Its condition can be seen obviously when $R_{e}=5.64 \times 10^{5}$ as shown in Fig. 4(b). In term of horizontal velocity distribution, Case 2 has different behavior with Case 1 and it is indicating that flow regime already move to higher flow regime. Therefore, Case 2 is categorized in weakly turbulent flow.

It can be summarized that present experiments are classified in two types flow regime, Case $1 ; R_{e}=$ $2.25 \times 10^{5}$ as laminar flow regime and the other three cases in transition to turbulence flow regime. Case 2 with $R_{e}=5.64 \times 10^{5}$ is categorized as weakly turbulent flow, while Case $3 ; R_{e}=6.06 \times 10^{5}$ and Case $4 ; R_{e}=7.34 \times 10^{5}$ are in conditionally turbulent flow. 

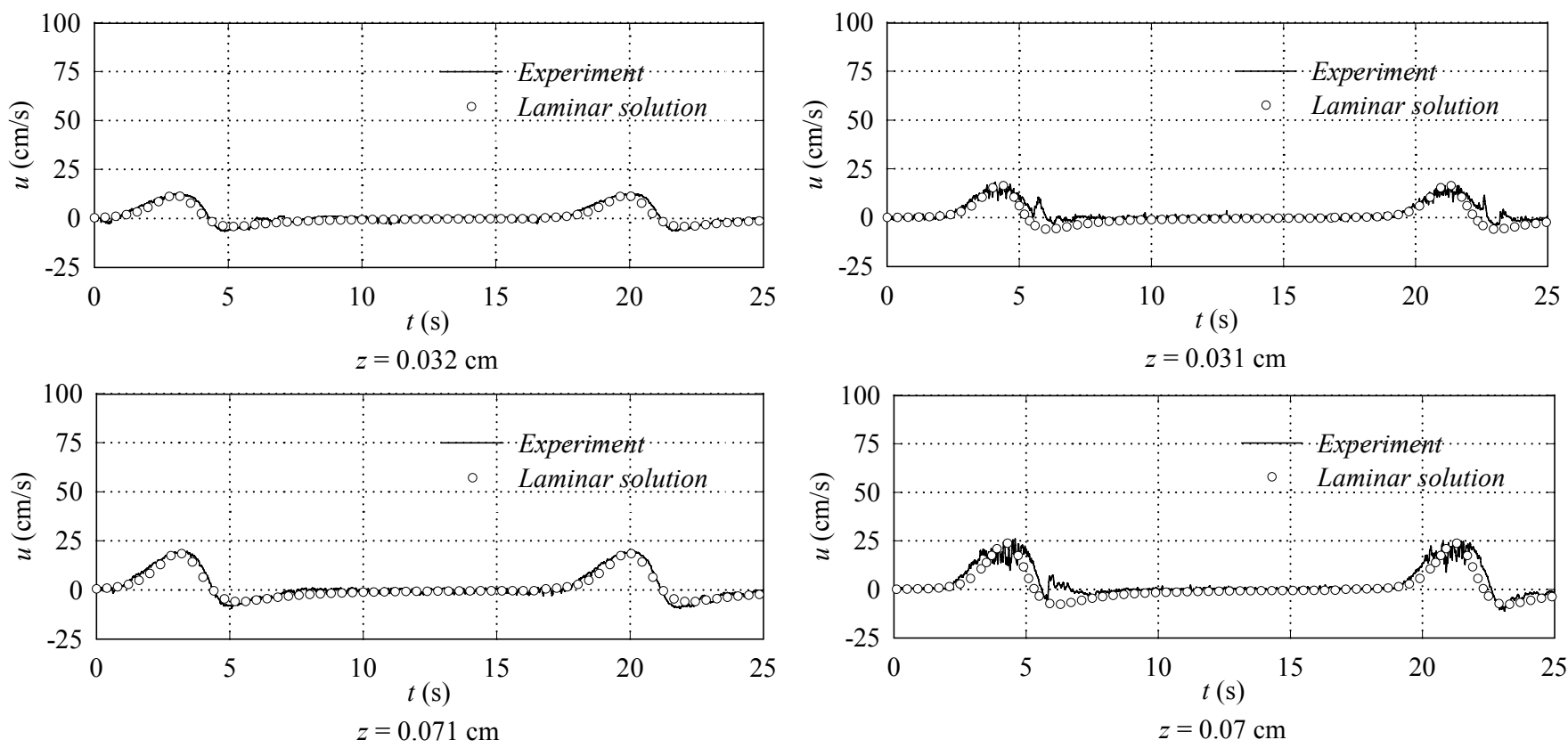

(a) Case $1\left(R_{e}=2.25 \times 10^{5}\right)$

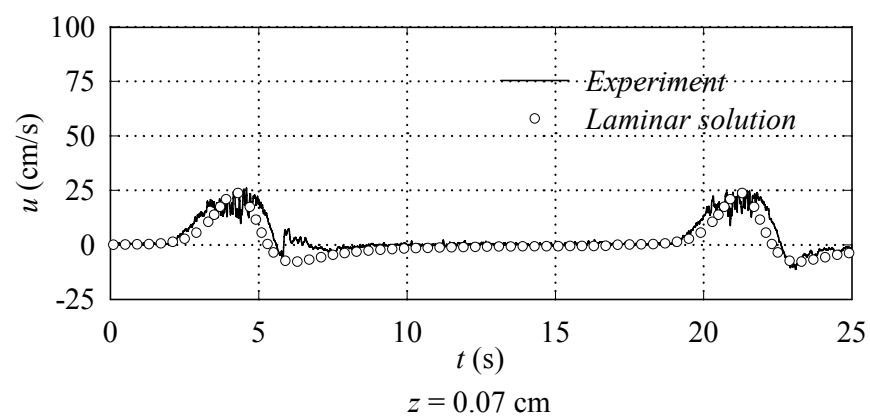

(b) Case $2\left(R_{e}=5.64 \times 10^{5}\right)$
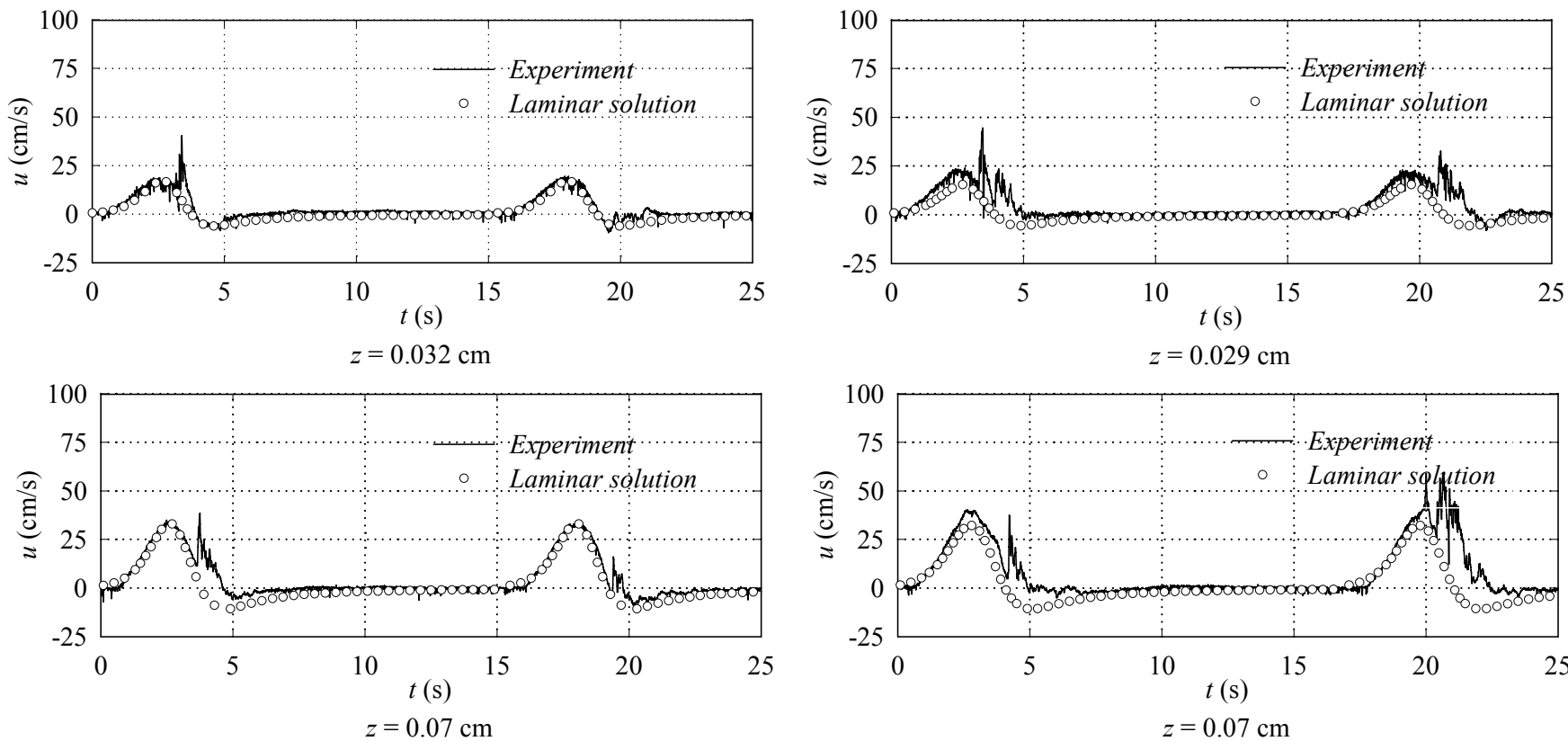

(c) Case $3\left(R_{e}=6.06 \times 10^{5}\right)$

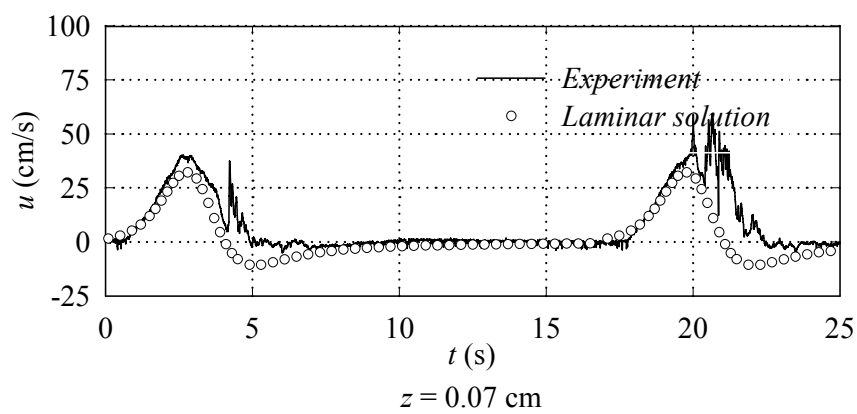

(d) Case $4\left(R_{e}=7.34 \times 10^{5}\right)$

Fig. 4 Time variation of velocity

Figure 5 illustrates vertical velocity distributions obtained from present experiment are compared with analytical solution for laminar flow. Figure 5(a) confirms that the experimental velocity distribution coincide well with the analytical solution for laminar flow during acceleration and deceleration phase (Case $1 ; R_{e}=2.25 \times 10^{5}$ ). And then starting deviation phenomenon from the laminar solution can be seen clearly when $R_{e}=$ $5.64 \times 10^{5}$ in deceleration phase (Fig. 5(b)). At $R_{e}=$ $7.34 \times 10^{5}$, the vertical velocity distribution diverge abruptly from the theoretical prediction for laminar flow (Fig. 5(c)) especially during deceleration phase.
The critical Reynolds number thus obtained is plotted in a stability diagram (Fig. 6) proposed by Sumer et al. ${ }^{4}$ along with numerical simulation result from $\mathrm{DNS}^{9)}$ and experimental result ${ }^{4)}$. In this figure, $h$ is the water depth or half of the closed conduit tunnel height and $\delta_{l}$ is conventional thickness equal to $(2 v / \alpha)^{1 / 2}$. The present experiment of Case $2\left(R_{e}=\right.$ $\left.5.64 \times 10^{5}\right)$ is in slightly higher than critical Reynolds number line at $R_{e} \approx 5 \times 10^{5}$. According to validation result Case 2 is categorized in transition to turbulence regime with weakly turbulent flow classification and it seems to be in good agreement with previous studies ${ }^{499}$. 


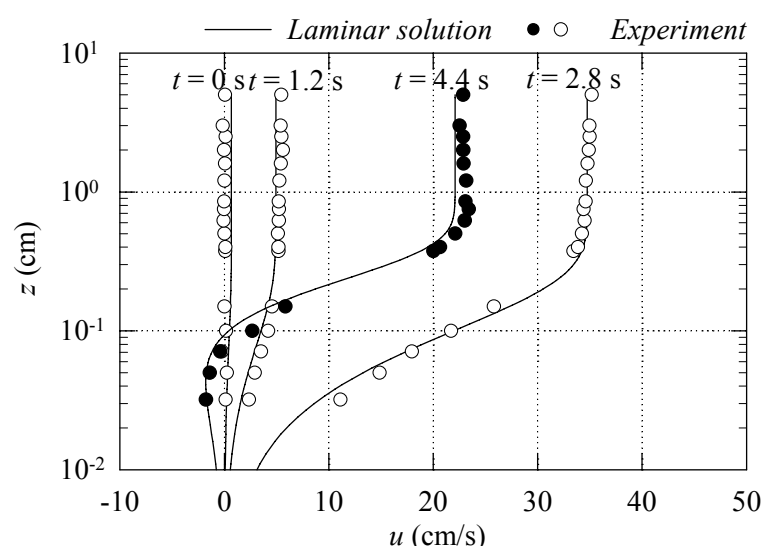

(a) Case $1\left(R_{e}=2.25 \times 10^{5}\right)$

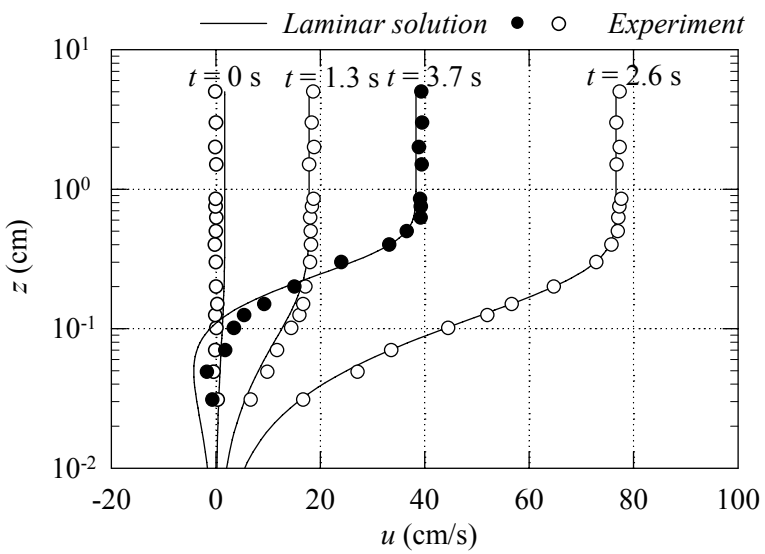

(b) Case $2\left(R_{e}=5.64 \times 10^{5}\right)$

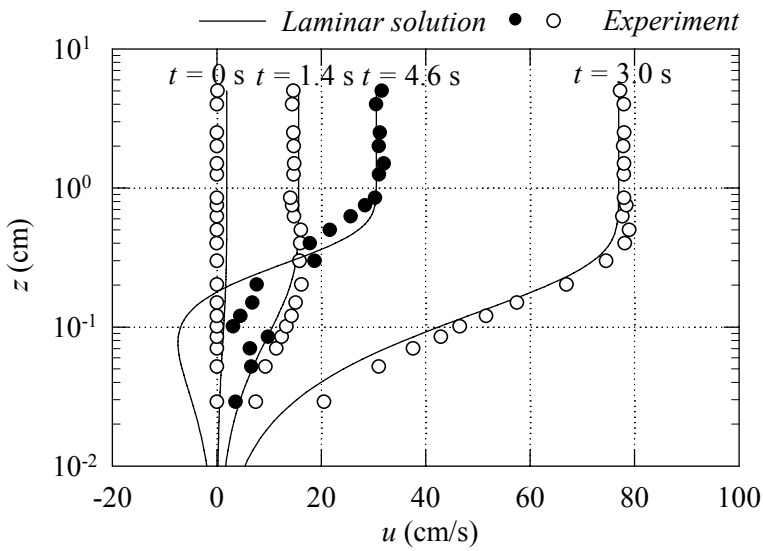

(c) Case $4\left(R_{e}=7.34 \times 10^{5}\right)$

Fig. 5 Vertical velocity distribution

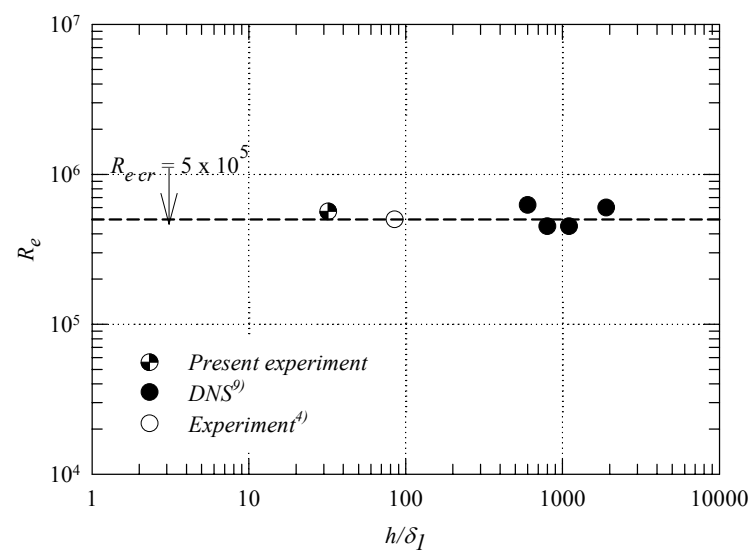

Fig. 6 Stability diagram

\section{CONCLUSIONS}

A new generation system for solitary wave boundary layer has been applied in laboratory experiment for various values of Reynolds number. Furthermore validations have been done in terms of free stream velocity, single and periodical oscillatory motion experiment and also time variation of velocity distribution. Two types of flow regime have been detected: laminar flow regime and transition to turbulence flow regime with weakly turbulent flow and conditionally turbulent flow. The critical Reynolds number obtained from this study shows good agreement with the finding of previous researchers.

\section{ACKNOWLEDGMENT}

This research was partially supported by Open Fund for Scientific Research from State Key Laboratory of Hydraulics and Mountain River Engineering, Sichuan University. The support from Hitachi Scholarship Foundation (HSF) for research activity of BW is also gratefully acknowledged.

\section{REFERENCES}

1) Liu, P. L. -F., Park, Y. S. and Cowen, E. A.: Boundary layer flow and bed shear stress under solitary wave, J. Fluid Mechanics., Vol. 574, pp.449-463, 2007.

2) Konishi, E., Suntoyo., Tanaka, H. and Yamaji, H: Study on bottom boundary layer under solitary wave motion, $J$. Applied Mech., Vol.10, pp.741-748, 2007. (in Japaese)

3) Yamaji, H., Tanaka, H., Suntoyo. and Tanaka, S.: Generation method of bottom boundary layer flow induced by solitary wave, J. Applied Mech., Vol.11, pp.835-842, 2008. (in Japanese)

4) Sumer, B. M., Jensen, P. M., Sorensen, L. B., Fredsoe, J., Liu, P. L. -F. and Cartesen, S: Coherent structures in wave boundary layers. Part 2 Solitary motion, J. Fluid Mechanics, Vol.646, pp.207-231, 2010.

5) Keulegan, G. H.: Gradual damping of solitary wave, U.S. Department of Commerce, National Bureau of Standards. RP1895, Vol. 40, pp.487-498, 1972.

6) Tanaka, H., Sumer, B. M. and Lodahl, C: Theoretical and experimental investigation on laminar boundary layer under cnoidal wave motion, Coastal Eng. J, Vol.40(1), pp.81-98, 1998.

7) Suntoyo. and Tanaka, H.: Numerical modeling of boundary layer flows for solitary wave, J. Hydro-Enviro. Res., Vol.3(3), pp.129-137, 2009.

8) Hino, M., Sawamoto, M. and Takasu, S: Experiments on transition to turbulence in an oscillatory pipe flow, J. Fluid Mechanics, Vol.75, pp.193-207, 1976.

9) Vittori, G. and Blondeaux, P.: Turbulent boundary layer under solitary wave, J. Fluid Mechanics, Vol.615, pp.433-443, 2008.

(Received September 30, 2010) 